\title{
UNIFORMIDADE DE DISTRIBUIÇÃO SUPERFICIAL E SUBSUPERFICIAL DE ÁGUA E DE ÁGUA RESIDUÁRIA DE SUINOCULTURA COM IRRIGAÇÃO POR ASPERSÃO EM MALHA ${ }^{1}$
}

\author{
LUÍS C. D. DRUMOND ${ }^{2}$, JOSÉ R. ZANINI ${ }^{3}$, ANDRÉ L. T. FERNANDES ${ }^{4}$, \\ GUILHERME P. RODRIGUES ${ }^{5}$
}

\begin{abstract}
RESUMO: A irrigação por aspersão em malha está sendo bastante utilizada em café, pastagem, cana forrageira e capineiras, por ser sistema de baixo custo, de fácil instalação e manejo e por permitir fertirrigação com água residuária de suinocultura e de bovinocultura. Para determinar a uniformidade de distribuição de água e de água residuária de suinocultura nesse sistema de irrigação, foi conduzido um experimento na Fazenda-Escola da Universidade de Uberaba, empregando-se pluviômetros eqüidistantes, entre quatro aspersores de duas linhas laterais. $\mathrm{O}$ coeficiente de uniformidade de Christiansen (CUC) e o coeficiente de uniformidade de distribuição (CUD) foram superiores aos valores mínimos recomendados, mesmo com $200 \mathrm{~m}^{3}$ de água residuária de suinocultura por hectare por ano.
\end{abstract}

PALAVRAS-CHAVE: água residuária, tubos enterrados, coeficiente de uniformidade.

\section{SUPERFICIAL AND SUBSUPERFICIAL DISTRIBUTION UNIFORMITY OF WATER AND WASTEWATER OF SWINE APPLIED BY NET-SPRINKLER IRRIGATION SYSTEM}

\begin{abstract}
The net-sprinkler irrigation is frequently being used in coffee, pasture, sugar cane forage and forages, due low cost system, easy installation and handling, allowing fertigation with wastewater of swine and bovine. Aiming to determine the water and wastewater of swine distribution uniformity with this irrigation system, an experiment was carried out at Uberaba University - Brazil, being used pluviometers among four sprinklers of two lateral lines. The Christiansen's uniformity coefficient (CUC) and the uniformity distribution coefficient (UDC) were superior to the minimum recommended values, even with $200 \mathrm{~m}^{3} \mathrm{ha}^{-1}$ year ${ }^{-1}$ of swine wastewater.
\end{abstract}

KEYWORDS: wastewater, subsurface pipes, uniformity coefficient.

\section{INTRODUÇÃO}

Conforme WOODWARD (1959), a introdução de fertilizantes orgânicos e inorgânicos em água de irrigação é praticada há séculos. Uma das principais aplicações por aspersão começou com adubos líquidos em pastagens.

CHRISTIANSEN (1942) foi o primeiro pesquisador que estudou a uniformidade de distribuição da água para aspersores rotativos, determinando o efeito da pressão de serviço, do espaçamento, da rotação e da velocidade do vento sobre a distribuição da água; estabeleceu o parâmetro conhecido como coeficiente de uniformidade de Christiansen (CUC). O SOIL CONSERVATION SERVICE (1968) propôs a equação para cálculo do coeficiente de uniformidade de distribuição (CUD), que considera a média dos $25 \%$ menores valores de precipitação em relação à média total.

\footnotetext{
${ }^{1}$ Extraído da tese de doutorado do primeiro autor.

${ }^{2}$ Eng $^{\circ}$ Agrônomo, Prof. Doutor, FAZU e UNIUBE, Uberaba - MG, Fone: (0XX34) 3318.4188, ldrumond@ fazu.br

${ }^{3}$ Eng $^{\mathrm{o}}$ Agrônomo, Prof. Doutor, Departamento de Engenharia Rural, UNESP, Jaboticabal - SP.

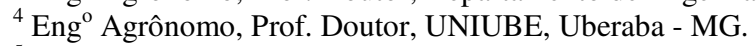

${ }^{5}$ Eng $^{\mathrm{o}}$ de Alimentos, Prof. M.Sc., UNIUBE, Uberaba - MG.

Recebido pelo Conselho Editorial em: 24-5-2005
}

Aprovado pelo Conselho Editorial em: 19-9-2006 
FRIZZONE (1992) cita que o coeficiente de uniformidade expressa a variabilidade da lâmina de irrigação aplicada na superfície do solo em relação à lâmina média aplicada, por meio de medidas de dispersão expressas na forma adimensional. A baixa eficiência de aplicação da irrigação por aspersão está relacionada com a não-uniformidade de distribuição de água e com as perdas de água por evaporação e arrastamento pelo vento.

MERRIAN \& KELLER (1978) afirmam que, para culturas de alto rendimento econômico, com sistema radicular raso, a irrigação por aspersão deve apresentar alta uniformidade de distribuição. O CUD deve ser superior a $80 \%$ e o CUC acima de $88 \%$. Para cultivos com sistema radicular médio, o CUD pode variar entre 70 e $80 \%$ e o CUC entre 80 e $88 \%$. Em culturas com sistema radicular profundo, a uniformidade de distribuição pode variar entre 50 e $70 \%$ e o CUC entre 70 e $80 \%$.

Trabalhos de modelagem e simulação computacional têm mostrado que fatores como temperatura e tamanho de gotas de água, temperatura e umidade relativa do ar, altura de instalação do aspersor e velocidade do vento, exercem efeito pronunciado na evaporação da água e na uniformidade de distribuição (SILVA, 1989). Segundo PEREIRA (1995), a utilização de modelos matemáticos que simulam a irrigação por aspersão é uma alternativa para estudar as interações desses diversos fatores e verificar a influência dos elementos climáticos nas perdas e na uniformidade do sistema.

Em muitas fazendas no Brasil, existe considerável volume de água residuária que poderia ser utilizada para adubação em várias culturas. Os custos com transporte e mão-de-obra para aplicação desses dejetos têm levado a se buscarem alternativas mais econômicas, como a aplicação via sistema de irrigação, pois, dependendo de sua origem, o adubo animal pode conter 60 a $98 \%$ de líquido. Desta forma, a aplicação de esterco líquido com sistema de irrigação é uma recomendável alternativa de reciclagem, considerando um sistema hidraulicamente bem projetado e operado de modo correto. Nos Estados Unidos, o uso da irrigação para aplicação de estercos líquidos apresenta tendência de crescimento desde o início da década de 1970.

Devido ao crescente interesse em implantar sistemas de aspersão em malha (DRUMOND \& FERNANDES, 2001) e realizar fertirrigação aplicando água residuária de suinocultura, no presente estudo, foram avaliados coeficientes de uniformidade de aplicação de água residuária de suinocultura (ARS) e de água, sobre e sob a superfície do solo.

\section{MATERIAL E MÉTODOS}

A pesquisa foi conduzida na Fazenda Alexandre Barbosa, da Universidade de Uberaba - MG, localizada a $19^{\circ} 45^{\prime}$ de latitude sul e a $47^{\circ} 55^{\prime}$ de longitude oeste, com altitude média de $840 \mathrm{~m}$. $\mathrm{O}$ experimento foi conduzido de março a agosto de 2000, numa área de 4,0 hectares, topografia suave ondulada, cultivada com capim Cynodon $s p$ cv Tifton 85, ocupada com vacas leiteiras colocadas quando a altura do capim era de 20 a $25 \mathrm{~cm}$ e retiradas quando a altura era de 10 a $12 \mathrm{~cm}$. As características físicas do solo são apresentadas na Tabela 1.

TABELA 1. Análise física do solo da área experimental.

\begin{tabular}{ccccc}
\hline Camada $(\mathrm{cm})$ & Areia $(\%)$ & Silte $(\%)$ & Argila $(\%)$ & Densidade aparente $\left(\mathrm{kg} \mathrm{dm}^{-3}\right)$ \\
\hline $0-20$ & 68,6 & 21,4 & 10,0 & 1,65 \\
$20-40$ & 68,9 & 21,6 & 9,5 & 1,58 \\
$40-60$ & 68,7 & 21,2 & 10,1 & 1,69 \\
\hline
\end{tabular}

\section{Sistema de irrigação}

Foi instalado um sistema de aspersão utilizando-se do aspersor Naan 5035, bocais 5,0 x 2,5 mm, pressão de serviço $280 \mathrm{kPa}$, vazão nominal de $1.875 \mathrm{~L} \mathrm{~h}^{-1}$ e ângulo de inclinação do jato igual a $23^{\circ}$, denominado aspersor A e o aspersor Netafim, modelo N 95, bocais 5,0 x 2,2 mm, pressão de serviço $280 \mathrm{kPa}$, vazão nominal de $1.685 \mathrm{~L} \mathrm{~h}^{-1}$, ângulo de inclinação do jato igual a $23^{\circ}$, 
denominado aspersor $\mathrm{B}$, instalados no espaçamento de 18 × 18 metros. O sistema utilizado foi semiportátil e de baixo custo, isto é, linhas principais, de derivação, e laterais fixas e enterradas, com mudança apenas dos aspersores, instalados a $60 \mathrm{~cm}$ de altura em relação ao solo, constituindo uma rede malhada, comumente denominada de aspersão em malha (DRUMOND e FERNANDES, 2001). As linhas laterais foram de PVC soldável, espaçadas de 18 metros, interligadas, pressão nominal 60 mca (PN 60; $588 \mathrm{kPa}$ ) e diâmetro de $25 \mathrm{~mm}$.

A linha de derivação ou secundária era de PVC soldável, de $50 \mathrm{~mm}$, PN 80, e a linha principal era também de PVC soldável, de $75 \mathrm{~mm}$ e PN 80 (Figura 1). As linhas laterais foram interligadas em anéis, e a estabilização hidráulica foi realizada utilizando o processo Hardy Cross (PORTO, 2000). Com isso, conseguiu-se operar com baixos diâmetros nas linhas laterais, com baixa potência do conjunto motobomba e, conseqüentemente, com economia de energia.

O manejo da umidade do solo foi realizado com aplicação de água residuária de suinocultura e água, baseando-se no balanço hídrico climatológico, com dados de clima obtidos em estação meteorológica automatizada Micrometos 300 e na retenção de água no solo (Tabela 2).

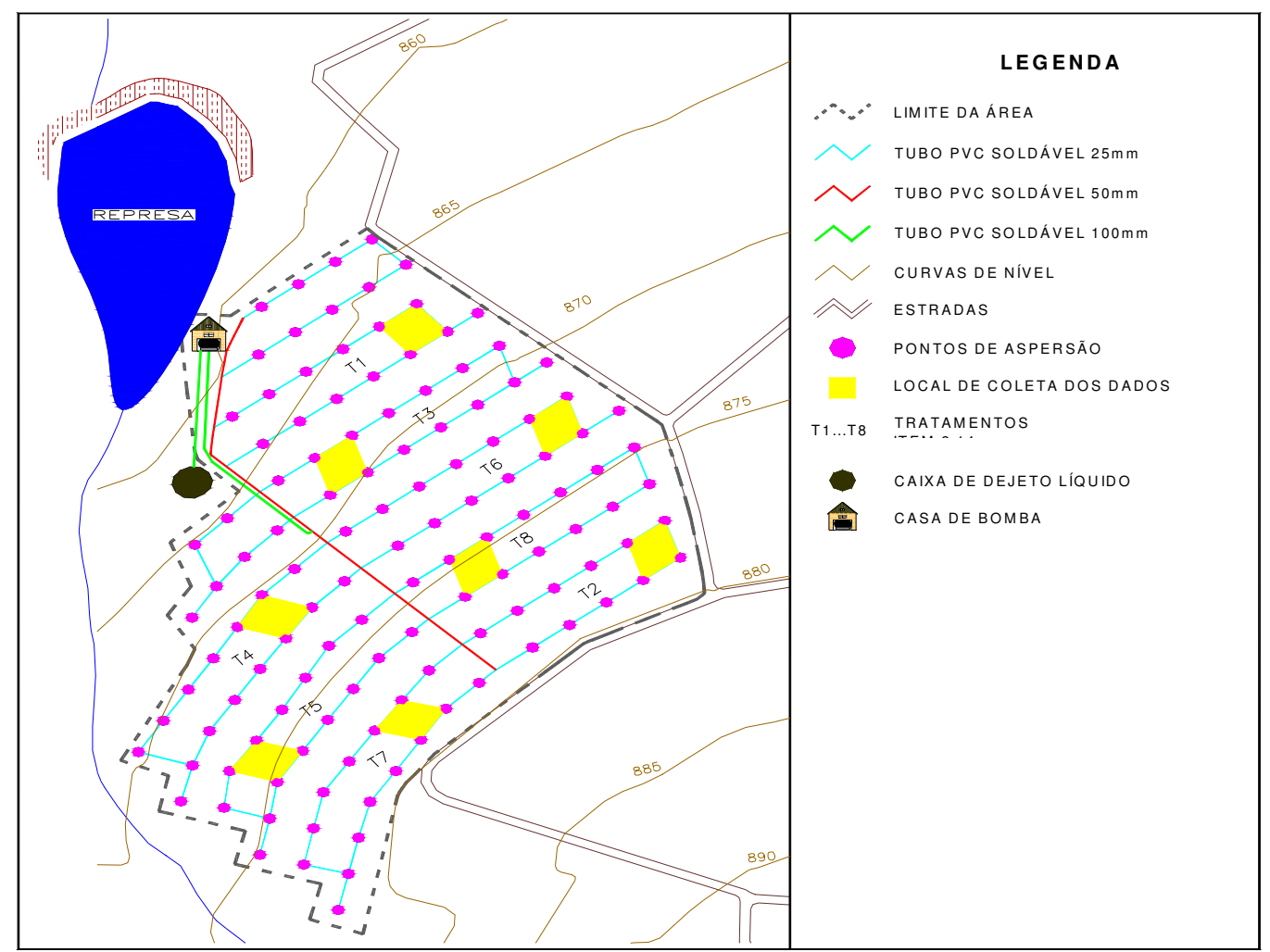

FIGURA 1. Planta plani-altimétrica da área experimental com irrigação em malha.

TABELA 2. Valores de retenção de água no solo para diferentes potenciais matriciais.

\begin{tabular}{cccccccccccc}
\hline \multirow{2}{*}{$\begin{array}{c}\text { Prof. } \\
(\mathrm{cm})\end{array}$} & 0 & 0,001 & 0,002 & 0,004 & 0,006 & 0,02 & 0,05 & 0,1 & 0,5 & 1,5 \\
\cline { 2 - 11 } & \multicolumn{10}{c}{$\mathrm{cm}^{3}$} & de água por cm $\mathrm{cm}^{3}$ de solo \\
\hline $0-20$ & 0,446 & 0,399 & 0,392 & 0,384 & 0,383 & 0,282 & 0,248 & 0,227 & 0,201 & 0,185 \\
$20-40$ & 0,462 & 0,414 & 0,402 & 0,394 & 0,392 & 0,312 & 0,267 & 0,245 & 0,232 & 0,206 \\
$40-60$ & 0,414 & 0,371 & 0,368 & 0,365 & 0,364 & 0,257 & 0,219 & 0,194 & 0,177 & 0,157 \\
\hline
\end{tabular}

Para que não houvesse déficit hídrico na área experimental, as lâminas aplicadas foram calculadas com base na evapotranspiração do dia anterior, estimada pela equação de PenmanMonteith, com adoção do turno de rega variável. 


\section{Água residuária de suinocultura (ARS)}

A água residuária foi obtida de uma granja de sistema de engorda, localizada próxima à área experimental. Os dejetos foram canalizados das baias da suinocultura, para uma caixa principal de separação. A função dessa caixa era separar o líquido do sólido e direcioná-lo para uma caixa secundária que, por sua vez, descarregava nas lagoas de estabilização. Após completar o período de tratamento nas lagoas, a ARS era conduzida a uma caixa de recepção com $4,6 \mathrm{~m}^{3}$, e uma tubulação de PVC ligava essa caixa à sucção da bomba. Ainda nessa caixa, havia uma tela para retenção de pêlos, visando a diminuir o entupimento dos bocais dos aspersores. $\mathrm{O}$ esquema do processo de separação da ARS até a lagoa de estabilização está representado na Figura 2.

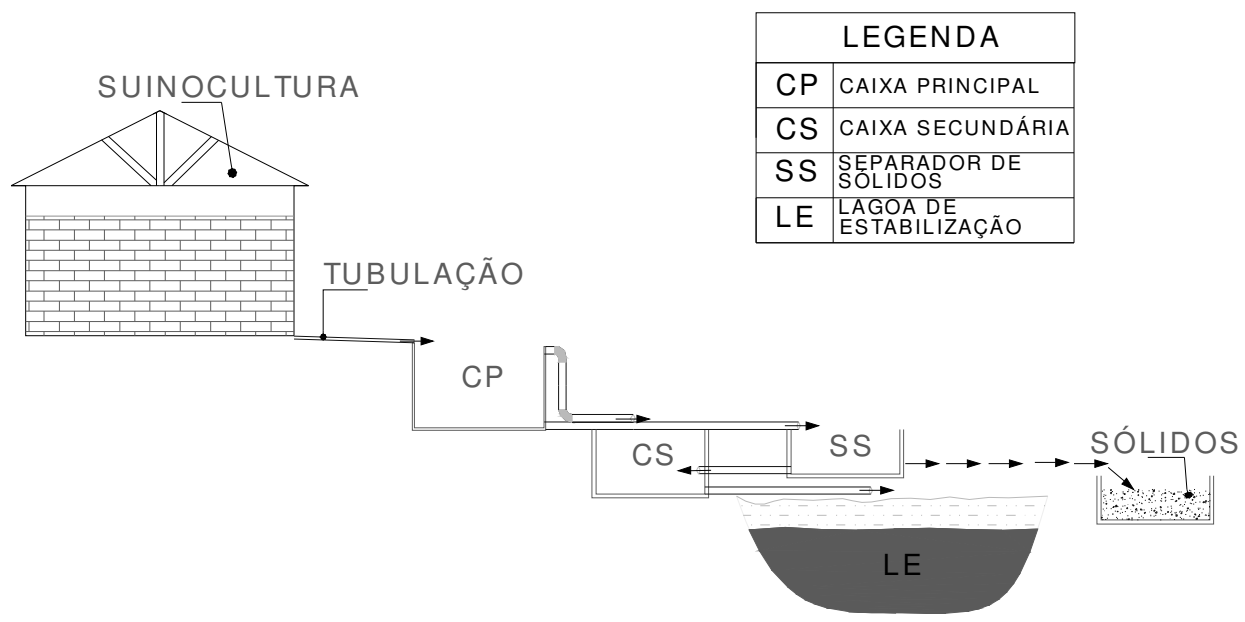

FIGURA 2. Esquema representando o processo de tratamento do dejeto.

Durante as aplicações, procurou-se manter constante a taxa de injeção de ARS no sistema de irrigação, e o controle do volume aplicado foi realizado na caixa de recepção, já que suas dimensões eram conhecidas. Uma tubulação de PVC ligava a caixa de recepção de ARS à sucção da motobomba, controlando-se a vazão da ARS com registro de gaveta. Assim, foi possível controlar a aplicação de ARS, para que tivesse a mesma duração do teste hidráulico, ou seja, de duas horas. Durante a aplicação de água, esse registro era mantido fechado.

No período de condução do experimento, foram realizadas três análises químicas da água e de ARS no Laboratório de Solos da Faculdade de Agronomia e Zootecnia de Uberaba (Tabelas 3 a $5)$.

TABELA 3. Caracterização química da ARS relativa à coleta realizada em 2 de março de 2000.

\begin{tabular}{lcccccccccccccc}
\hline \multirow{2}{*}{ Elementos } & $\mathrm{N}$ & $\mathrm{P}$ & $\mathrm{K}$ & $\mathrm{Ca}$ & $\mathrm{S}$ & $\mathrm{Mg}$ & $\mathrm{Zn}$ & $\mathrm{Cu}$ & $\mathrm{B}$ & $\mathrm{Fe}$ & $\mathrm{Mn}$ & $\mathrm{Na}^{2}$ & $\mathrm{MO}^{1}$ & $\mathrm{Dens}^{2}$ \\
\cline { 2 - 17 } & $\%$ & $\%$ & $\mathrm{ppm}$ & $\mathrm{ppm}$ & $\mathrm{ppm}$ & $\mathrm{ppm}$ & $\mathrm{ppm}$ & $\mathrm{ppm}$ & $\mathrm{ppm}$ & $\mathrm{ppm}$ & $\mathrm{ppm}$ & $\mathrm{ppm}$ & $\%$ & $\mathrm{~g} \mathrm{~mL}^{-1}$ \\
\hline \multirow{2}{*}{ Água } & - & - & 0,17 & 0,17 & - & 0,03 & 0,6 & - & - & 1,9 & - & 0,3 & - & 1,02 \\
ARS & 1,60 & 0,19 & 620,0 & 100,0 & 50,0 & 50,0 & 5,7 & 4,0 & 6,0 & 25,0 & 5,0 & 142,0 & 0,40 & 0,97 \\
\hline
\end{tabular}

(1) MO: matéria orgânica; (2) Dens: densidade da amostra.

TABELA 4. Caracterização química da ARS relativa à coleta realizada em 28 de abril de 2000.

\begin{tabular}{lcccccccccccccc}
\hline \multirow{2}{*}{ Elementos } & $\mathrm{N}$ & $\mathrm{P}$ & $\mathrm{K}$ & $\mathrm{Ca}$ & $\mathrm{S}$ & $\mathrm{Mg}$ & $\mathrm{Zn}$ & $\mathrm{Cu}$ & $\mathrm{B}$ & $\mathrm{Fe}$ & $\mathrm{Mn}$ & $\mathrm{Na}^{2}$ & $\mathrm{MO}^{1}$ & $\mathrm{Dens}^{2}$ \\
\cline { 2 - 22 } & $\%$ & $\%$ & $\mathrm{ppm}$ & $\mathrm{ppm}$ & $\mathrm{ppm}$ & $\mathrm{ppm}$ & $\mathrm{ppm}$ & $\mathrm{ppm}$ & $\mathrm{ppm}$ & $\mathrm{ppm}$ & $\mathrm{ppm}$ & $\mathrm{ppm}$ & $\%$ & $\mathrm{~g} \mathrm{~mL}^{-1}$ \\
\hline Água & - & - & 0,13 & 0,18 & - & 0,03 & 0,6 & - & - & 2,2 & - & 0,3 & - & 1,00 \\
ARS & 1,28 & 0,18 & 580,0 & 100,0 & 50,0 & 50,0 & 6,0 & 4,0 & 6,0 & 25,0 & 5,0 & 140,0 & 0,34 & 0,97 \\
\hline
\end{tabular}

(1) MO: matéria orgânica; (2) Dens: densidade da amostra.

TABELA 5. Caracterização química da ARS relativa à coleta realizada em 20 de junho de 2000.

\begin{tabular}{|c|c|c|c|c|c|c|c|c|c|c|c|c|c|c|}
\hline \multirow{2}{*}{ Elementos } & $\mathrm{N}$ & $P$ & $\mathrm{~K}$ & $\mathrm{Ca}$ & $\mathrm{S}$ & $\mathrm{Mg}$ & $\mathrm{Zn}$ & $\mathrm{Cu}$ & B & $\mathrm{Fe}$ & $\mathrm{Mn}$ & $\mathrm{Na}$ & $\mathrm{MO}^{1}$ & Dens $^{2}$ \\
\hline & $\%$ & $\%$ & ppm & $\mathrm{ppm}$ & ppm & ppm & $\mathrm{ppm}$ & $\mathrm{ppm}$ & ppm & $\mathrm{ppm}$ & $\mathrm{ppm}$ & $\mathrm{ppm}$ & $\%$ & $\mathrm{~g} \mathrm{~mL}^{-1}$ \\
\hline Água & - & - & 0,15 & 0,19 & - & 0,03 & 0,6 & - & - & 2,0 & - & 0,3 & - & 1,01 \\
\hline ARS & 1,08 & 0,17 & 600,0 & 100,0 & 50,0 & 50,0 & 6,0 & 4,0 & 6,0 & 25,0 & 5,0 & 144,0 & 0,30 & 0,97 \\
\hline
\end{tabular}

(1) MO: matéria orgânica; (2) Dens: densidade da amostra. 


\section{Uniformidade de distribuição de água e de ARS}

Para determinar a uniformidade de distribuição de água e da ARS, foram instalados pluviômetros, eqüidistantes, entre quatro aspersores de duas linhas laterais (Figura 3). As determinações foram realizadas com quatro repetições, tendo-se sempre uma no início e outra no final da malha. As doses de ARS foram parceladas, de forma que foi realizada uma aplicação semanal dentro do período dos ciclos, constituindo as repetições.

Foram utilizados 36 coletores, com $0,65 \mathrm{~m}$ de altura em relação à superfície do solo, e a seção de coleta de cada coletor era de $75 \mathrm{~cm}^{2}$. O volume coletado em cada pluviômetro foi determinado ao final do ensaio, utilizando-se de proveta de vidro graduada a cada $0,20 \mathrm{~mL}$. Os dados de direção e velocidade do vento foram obtidos pela estação climatológica automatizada, instalada a $100 \mathrm{~m}$ do local dos testes. Sempre que, durante os testes, a velocidade do vento ultrapassava $2 \mathrm{~m} \mathrm{~s}^{-1}$, o ensaio era descartado.

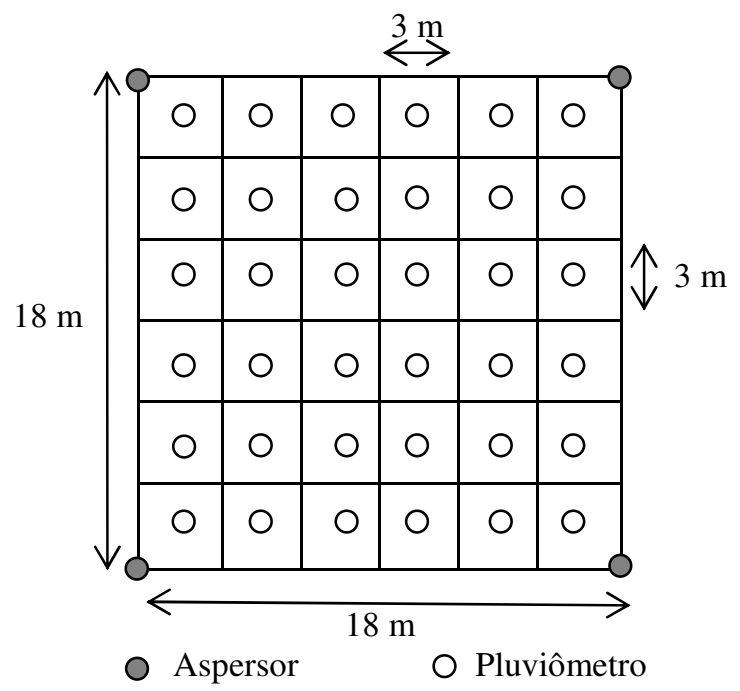

FIGURA 3. Disposição dos pluviômetros para determinar a distribuição de água e de ARS.

O CUC foi calculado aplicando-se a eq.(1) (CHRISTIANSEN, 1942) e, para o cálculo do CUD, aplicou-se a eq.(2) (SOIL CONSERVATION SERVICE, 1968).

$$
\mathrm{CUC}=100\left(1-\frac{\sum_{\mathrm{i}=1}^{\mathrm{n}}\left|X_{\mathrm{i}}-\mathrm{X}\right|}{\mathrm{nX}}\right)
$$

em que,

CUC - coeficiente de uniformidade de Christiansen, \%;

$\mathrm{X}_{\mathrm{i}}$ - precipitação no pluviômetro de ordem i, mm;

$\mathrm{X}$ - média aritmética das precipitações, $\mathrm{mm}$; e

$\mathrm{n}$ - número de pluviômetros.

$$
\mathrm{CUD}=\frac{\mathrm{x}}{\mathrm{X}} 100
$$

em que,

CUD - coeficiente de uniformidade de distribuição, \%, e

$\mathrm{x}$ - média de $25 \%$ do total de pluviômetros com as menores precipitações, $\mathrm{mm}$.

\section{Uniformidade de distribuição subsuperficial da água e da ARS}

A determinação da uniformidade de distribuição abaixo da superfície do solo foi realizada utilizando-se de amostras do solo, coletadas aleatoriamente nas quadrículas, utilizando-se de um trado a $20 \mathrm{~cm}$ de profundidade, obtendo-se uma amostra composta originada de 12 coletas, para cada uma das 36 quadrículas. As amostras foram coletadas 24 horas após o final da irrigação (RESENDE, 1992). Utilizou-se do método gravimétrico para determinar a umidade do solo, e a uniformidade foi calculada de forma idêntica à realizada acima da superfície. 


\section{Delineamento experimental e tratamentos utilizados}

Foi adotado o delineamento experimental em parcelas subdivididas. As parcelas representaram os aspersores, e as subparcelas, as doses de água e de dejeto líquido de suíno ( $0 ; 50$; 100 e $200 \mathrm{~m}^{3} \mathrm{ha}^{-1} \mathrm{ano}^{-1}$ ), constituindo um fatorial $2 \times 4$, com quatro repetições, que foram caracterizadas pelas aplicações semanais dentro do período dos ciclos. As doses estabelecidas de ARS e de água foram parceladas em 24 aplicações ao longo do experimento, em seis ciclos de irrigação, com quatro repetições por ciclo, avaliando-se oito tratamentos:

Tratamento 1 - Aplicação de água com aspersor A (T1);

Tratamento 2 - Aplicação de $50 \mathrm{~m}^{3} \mathrm{ha}^{-1} \mathrm{ano}^{-1}$ de ARS com aspersor A (T2);

Tratamento 3 - Aplicação de $100 \mathrm{~m}^{3} \mathrm{ha}^{-1} \mathrm{ano}^{-1}$ de ARS com aspersor A (T3);

Tratamento 4 - Aplicação de $200 \mathrm{~m}^{3} \mathrm{ha}^{-1} \mathrm{ano}^{-1}$ de ARS com aspersor A (T4);

Tratamento 5 - Aplicação de água com aspersor B (T5) ;

Tratamento 6 - Aplicação de $50 \mathrm{~m}^{3} \mathrm{ha}^{-1} \mathrm{ano}^{-1}$ de ARS com aspersor B (T6);

Tratamento 7 - Aplicação de $100 \mathrm{~m}^{3} \mathrm{ha}^{-1} \mathrm{ano}^{-1}$ de ARS com aspersor B (T7);

Tratamento 8 - Aplicação de $200 \mathrm{~m}^{3} \mathrm{ha}^{-1}$ ano $^{-1}$ de ARS com aspersor B (T8).

\section{RESULTADOS E DISCUSSÃO}

A uniformidade de distribuição é normalmente determinada pelo CUC e pelo CUD acima da superfície do solo. Neste experimento, o CUC foi sempre maior que o CUD acima da superfície do solo (Figuras 4 e 5). Esse fato é inerente às variáveis das equações utilizadas para se determinarem tais coeficientes, uma vez que, para o cálculo do CUD, são considerados os $25 \%$ da área que recebeu as menores lâminas. Resultados semelhantes foram encontrados por COTRIM (1988) e por RESENDE (1992), trabalhando com pivô central.

Pelas Figuras 4 e 5, verifica-se que, embora as equações ajustadas não permitam afirmar que os valores de CUC e CUD decresceram aumentando-se a dose de ARS de 150 para $200 \mathrm{~m}^{3} \mathrm{ha}^{-1}$ ano $^{-1}$, de modo geral, pode-se observar que esses coeficientes decresceram com o aumento da dose de ARS até $150 \mathrm{~m}^{3} \mathrm{há}^{-1}$ ano $^{-1}$. Esse comportamento indica que o entupimento dos bocais, devido principalmente à presença de pêlos, tem influência na uniformidade de aplicação. Porém, mesmo com as doses de 150 e $200 \mathrm{~m}^{3} \mathrm{ha}^{-1}$ ano $^{-1}$, os valores de CUC e CUD estiveram acima dos valores mínimos recomendados para irrigação por aspersão.

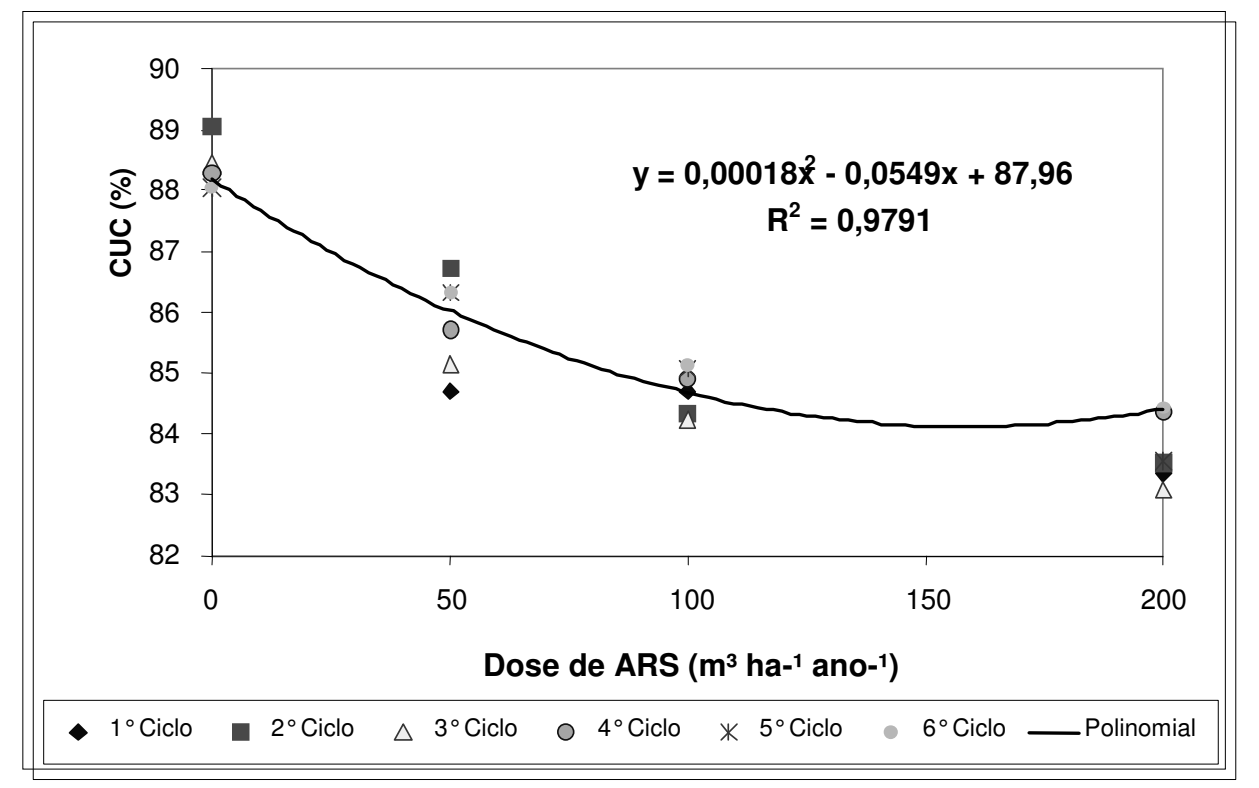

FIGURA 4. Valores de CUC acima da superfície do solo, de acordo com a dose de ARS. 


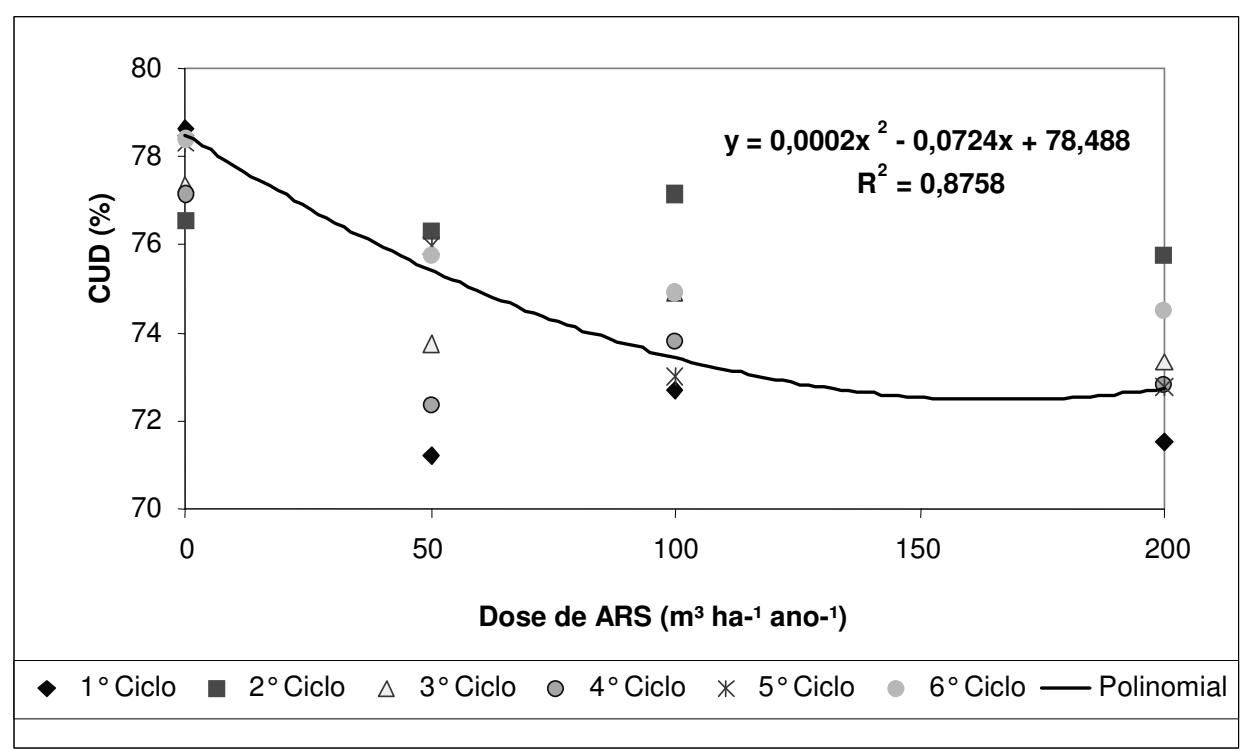

FIGURA 5. Valores de CUD acima da superfície do solo, de acordo com a dose de ARS.

Ocorreu boa correlação entre os valores de CUC para as doses de ARS, com $\mathrm{R}^{2}$ da ordem de 0,98. Quanto ao CUD, o coeficiente $\mathrm{R}^{2}$ foi menor, com valor médio de 0,88 , indicando maior variabilidade dos dados obtidos.

Os valores obtidos na análise de variância para os coeficientes CUC e CUD acima da superfície do solo encontram-se nas Tabelas 6 e 7, respectivamente. Pela Tabela 6, verifica-se que ocorreram diferenças significativas, entre os modelos dos aspersores em todos os ciclos, com exceção do $2^{\underline{0}}$ ciclo de coleta de dados. Embora os testes tenham sido realizados para velocidades de vento menores que $2 \mathrm{~m} \mathrm{~s}^{-1}$, por se tratar de um coeficiente que sofre grande influência desse fator, tal fato pode ser atribuído a condições pontuais ocorridas no momento dos testes, no $2^{0}$ ciclo. A interação não-significativa implica que os fatores modelo dos aspersores e dose de ARS atuaram de forma independente. Ocorreram diferenças significativas com relação à dose de ARS no CUC, exceto no $4^{\circ}$ ciclo de coleta de dados.

TABELA 6. Resultado da análise de variância para o CUC acima da superfície do solo.

\begin{tabular}{cccccccc}
\hline \multirow{2}{*}{ Fatores } & \multirow{2}{*}{$\mathrm{GL}$} & $1^{\mathrm{o}}$ Ciclo & $2^{-}$Ciclo & $3^{-}$Ciclo & $4^{-}$Ciclo & $5^{\circ}$ Ciclo & $6^{\circ}$ Ciclo \\
\cline { 3 - 8 } & & $\mathrm{F}$ & $\mathrm{F}$ & $\mathrm{F}$ & $\mathrm{F}$ & $\mathrm{F}$ & $\mathrm{F}$ \\
\hline Modelo dos Aspersores & 1 & $6,47^{*}$ & $0,02^{\mathrm{NS}}$ & $59,10^{*}$ & $8,69^{*}$ & $107,78^{*}$ & $92,33^{*}$ \\
Dose de ARS & 3 & $6,52^{*}$ & $9,25^{*}$ & $14,14^{*}$ & $2,44^{\mathrm{NS}}$ & $11,89^{*}$ & $10,02^{*}$ \\
Interação & 3 & $1,99^{\mathrm{NS}}$ & $1,59^{\mathrm{NS}}$ & $0,61^{\mathrm{NS}}$ & $0,21^{\mathrm{NS}}$ & $2,75^{\mathrm{NS}}$ & $1,30^{\mathrm{NS}}$ \\
\hline
\end{tabular}

* significativo a $5 \%$ de probabilidade; ${ }^{\text {NS }}$ não-significativo a $5 \%$ de probabilidade.

A análise de variância representada na Tabela 7 evidencia que ocorreram diferenças significativas no que se refere ao modelo dos aspersores, quanto ao CUD, exceto no primeiro e segundo ciclos de coleta de dados. Houve considerável variação entre as lâminas coletadas, ocorrendo grande influência nas médias das menores precipitações correspondentes a $25 \%$ da área, as quais são utilizadas para determinação desse parâmetro. A análise de variância foi significativa no primeiro, quinto e sexto ciclos de avaliação do experimento para a dose aplicada. De forma idêntica ao CUC, a interação dos fatores estudados para o CUD não foi significativa, sugerindo que os fatores atuaram de forma independente. 
TABELA 7. Resultado da análise de variância para o CUD acima da superfície do solo.

\begin{tabular}{cccccccc}
\hline \multirow{2}{*}{ Fatores } & \multirow{2}{*}{ GL } & $1^{0}$ Ciclo & $2^{0}$ Ciclo & $3^{\circ}$ Ciclo & $4^{-}$Ciclo & $5^{-}$Ciclo & $6^{\circ}$ Ciclo \\
\cline { 4 - 8 } & & $\mathrm{F}$ & $\mathrm{F}$ & $\mathrm{F}$ & $\mathrm{F}$ & $\mathrm{F}$ & $\mathrm{F}$ \\
\hline Modelo dos Aspersores & 1 & $1,78^{\mathrm{NS}}$ & $3,01^{\mathrm{NS}}$ & $23,71^{*}$ & $18,45^{*}$ & $34,50^{*}$ & $50,36^{*}$ \\
Dose de ARS & 3 & $5,44^{*}$ & $0,16^{\mathrm{NS}}$ & $1,99^{\mathrm{NS}}$ & $2,22^{\mathrm{NS}}$ & $12,26^{*}$ & $5,75^{*}$ \\
Interação & 3 & $2,52^{\mathrm{NS}}$ & $0,28^{\mathrm{NS}}$ & $0,56^{\mathrm{NS}}$ & $0,22^{\mathrm{NS}}$ & $1,29^{\mathrm{NS}}$ & $1,41^{\mathrm{NS}}$ \\
\hline
\end{tabular}

* significativo a $5 \%$ de probabilidade; ${ }^{\mathrm{NS}}$ não-significativo a $5 \%$ de probabilidade

Nas Figuras 6 e 7, são apresentadas as correlações entre os valores de CUCa e de CUDa (abaixo da superfície do solo), com a dose aplicada de ARS. Verifica-se que o comportamento foi semelhante ao obtido acima da superfície, isto é, os valores de CUC foram maiores que os valores de CUD. No entanto, abaixo da superfície do solo, houve aumento considerável da uniformidade de aplicação em todas as doses estudadas de ARS. Porém, mesmo com o processo de redistribuição da água no solo, pode-se observar que os valores de CUC e CUD decresceram com o aumento da dose de ARS, reproduzindo o comportamento desses coeficientes acima da superfície do solo. Isso pode ser explicado pela quantidade de sólidos na ARS ser em torno de $2 \%$ somente. Resultados semelhantes foram obtidos por HART (1972), TOOYAMANI et al. (1987) e RESENDE (1992). Trabalhando apenas com água, esses autores concluíram que existe redistribuição da água aplicada no solo, ocorrendo sensível acréscimo da uniformidade de aplicação. PAIVA (1980) também obteve redistribuição de água no perfil do solo, aplicada com sistema de aspersão convencional, com aumento nos coeficientes de uniformidade em todas as profundidades estudadas; mesmo nos ensaios onde a uniformidade na superfície foi baixa, em curtos espaços de tempo, os coeficientes de uniformidade alcançaram valores altos abaixo da superfície.

Na Figura 6, verifica-se que o coeficiente $\mathrm{R}^{2}$ foi da ordem de 0,95 para o CUCa. Pode-se considerar que ocorreu boa correlação entre os valores de CUCa para as doses aplicadas de ARS. Para o CUDa (Figura 7), o $\mathrm{R}^{2}$ foi menor, em torno de 0,88 , indicando maior variação dos valores obtidos. Essa maior variação do CUD em relação ao CUC, abaixo da superfície, aconteceu de forma semelhante ao obtido acima da superfície do solo.

Nas Tabelas 8 e 9, são apresentados os valores da análise de variância para os coeficientes CUC e CUD abaixo da superfície do solo. Pela Tabela 8, pode-se verificar que não houve efeito significativo do modelo dos aspersores, exceto no quinto ciclo, mostrando que, na maioria dos casos, não houve diferença de distribuição de água entre os modelos de aspersores. Isso ocorreu devido ao efeito da redistribuição da água abaixo da superfície do solo. Quando se observa a dose de ARS, verifica-se efeito significativo em todos os ciclos, indicando que a dose aplicada interferiu na uniformidade de distribuição. Com exceção do primeiro ciclo de coleta de dados, a interação foi não-significativa.

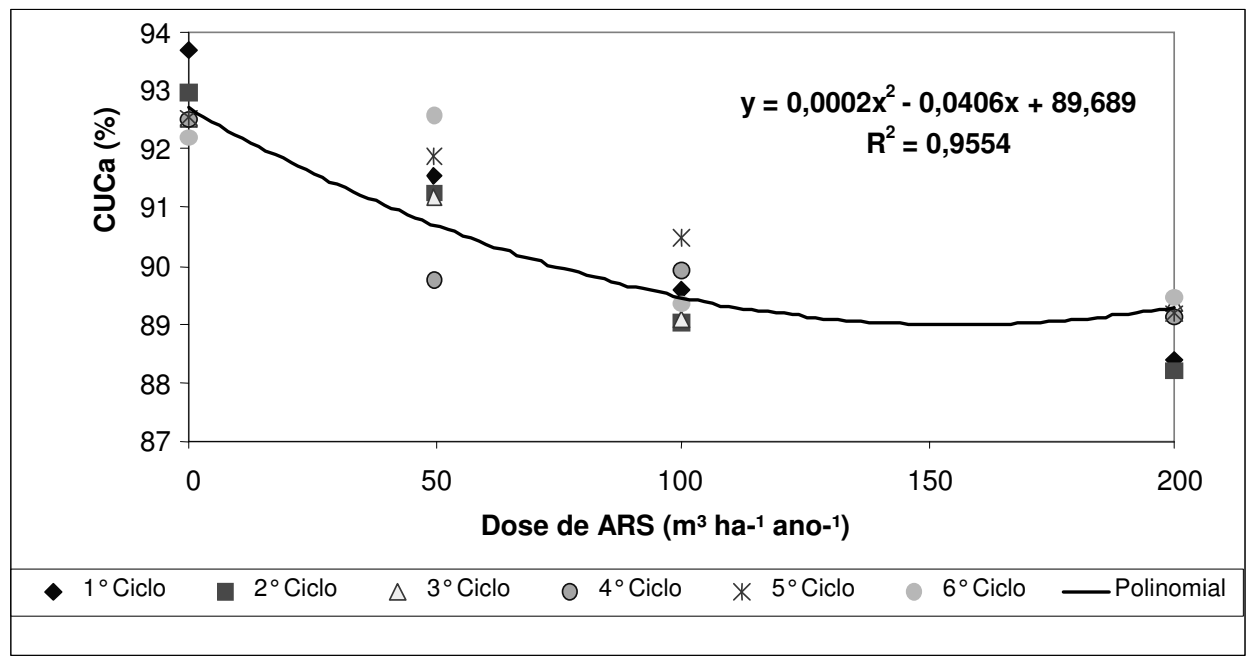

FIGURA 6. Valores de CUCa, de acordo com a dose de ARS. 


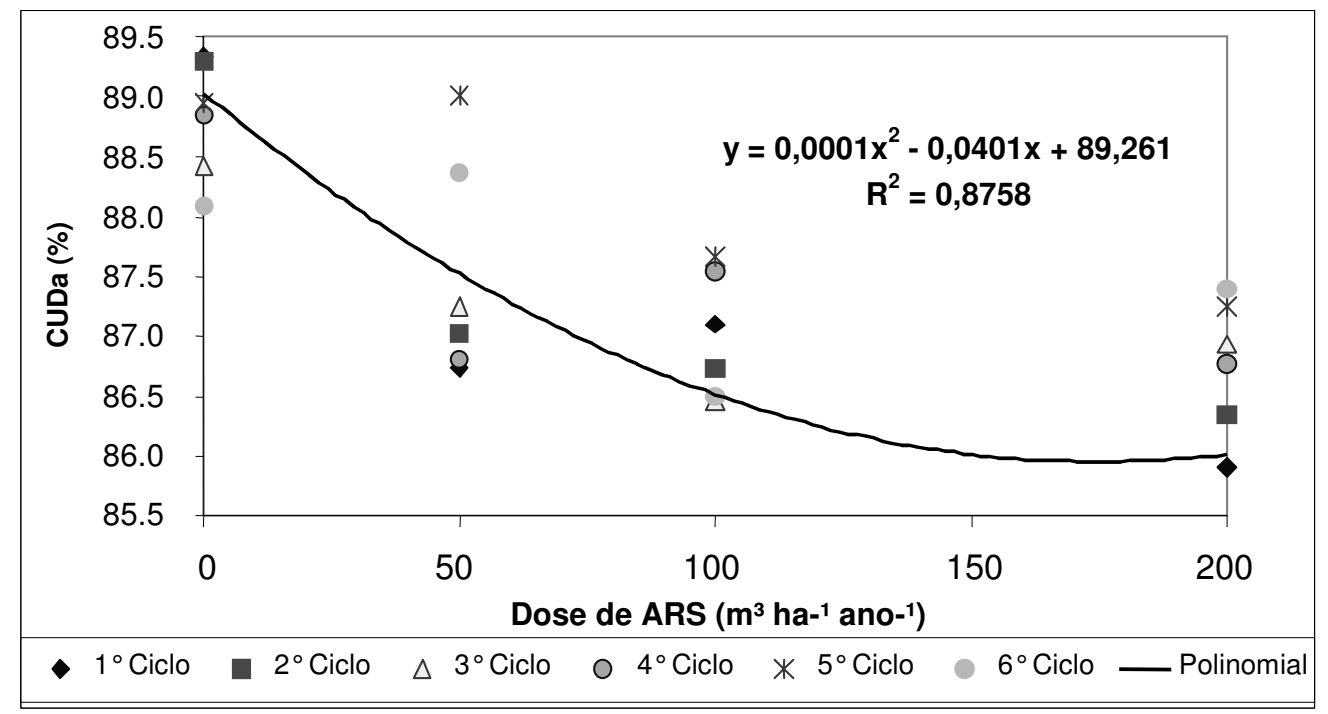

FIGURA 7. Valores de CUDa, de acordo com a dose de ARS.

TABELA 8. Resultado da análise de variância para o CUCa.

\begin{tabular}{cccccccc}
\hline \multirow{2}{*}{ Fatores } & \multirow{2}{*}{$\mathrm{GL}$} & $1^{\circ}$ Ciclo & $2^{-}$Ciclo & $3^{\circ}$ Ciclo & $4^{\circ}$ Ciclo & $5^{\circ}$ Ciclo & $6^{\circ}$ Ciclo \\
\cline { 3 - 7 } & & $\mathrm{F}$ & $\mathrm{F}$ & $\mathrm{F}$ & $\mathrm{F}$ & $\mathrm{F}$ & $\mathrm{F}$ \\
\hline Modelo dos Aspersores & 1 & $0,09^{\mathrm{NS}}$ & $0,01^{\mathrm{NS}}$ & $0,93^{\mathrm{NS}}$ & $0,24^{\mathrm{NS}}$ & $13,12^{*}$ & $1,89^{\mathrm{NS}}$ \\
Dose de ARS & 3 & $12,74^{*}$ & $15,76^{*}$ & $8,66^{*}$ & $4,32^{*}$ & $9,29^{*}$ & $13,07^{*}$ \\
Interação & 3 & $5,58^{*}$ & $1,78^{\mathrm{NS}}$ & $1,51^{\mathrm{NS}}$ & $0,40^{\mathrm{NS}}$ & $0,71^{\mathrm{NS}}$ & $0,57^{\mathrm{NS}}$ \\
\hline
\end{tabular}

* significativo a de $5 \%$ de probabilidade; ${ }^{\text {NS }}$ não-significativo a $5 \%$ de probabilidade

TABELA 9. Resultado da análise de variância para o CUDa.

\begin{tabular}{cccccccc}
\hline \multirow{2}{*}{ Fatores } & \multirow{2}{*}{$\mathrm{GL}$} & $1^{-\mathrm{o}}$ Ciclo & $2^{-0}$ Ciclo & $3^{-}$Ciclo & $4^{-}$Ciclo & $5^{-}$Ciclo & $6^{-0}$ Ciclo \\
\cline { 3 - 8 } & & $\mathrm{F}$ & $\mathrm{F}$ & $\mathrm{F}$ & $\mathrm{F}$ & $\mathrm{F}$ & $\mathrm{F}$ \\
\hline Modelo dos Aspersores & 1 & $5,72^{*}$ & $2,06^{\mathrm{NS}}$ & $10,35^{*}$ & $2,66^{\mathrm{NS}}$ & $0,20^{\mathrm{NS}}$ & $0,06^{\mathrm{NS}}$ \\
Dose de ARS & 3 & $5,47^{*}$ & $5,63^{*}$ & $3,20^{*}$ & $2,65^{\mathrm{NS}}$ & $5,61^{*}$ & $4,55^{*}$ \\
Interação & 3 & $3,85^{*}$ & $3,48^{*}$ & $5,33^{*}$ & $1,27^{\mathrm{NS}}$ & $3,92^{*}$ & $2,36^{\mathrm{NS}}$ \\
\hline
\end{tabular}

* significativo a $5 \%$ de probabilidade; ${ }^{\text {NS }}$ não-significativo a $5 \%$ de probabilidade

Na Tabela 9, para modelo dos aspersores, verifica-se que ocorreram efeitos significativos apenas no primeiro e terceiro ciclos. Para a dose de ARS, verifica-se efeito significativo, exceto no quarto ciclo. Ocorreu considerável variação na interação, sendo significativa para o primeiro, segundo, terceiro e quinto ciclos e não-significativa no quarto e sexto ciclos.

A comparação das médias dos tratamentos para CUC e CUD, segundo a metodologia de Tukey, pode ser observada na Tabela 10. Verifica-se que, tanto para o aspersor A, quanto para o aspersor $\mathrm{B}$, foram encontradas diferenças significativas entre os tratamentos. Os comportamentos do CUC e CUD foram bastante semelhantes, evidenciando que, para a maioria dos tratamentos, o aumento da dose de ARS provocou redução desses coeficientes, acima e abaixo da superfície do solo.

A influência do aumento da dose de ARS nos valores de CUC e CUD assemelha-se ao ocorrido com a vazão dos aspersores. Com o aumento da dose de ARS, ocorreu entupimento dos bocais dos aspersores, influenciando na uniformidade de aplicação. Nas condições em que o experimento foi realizado, os aspersores A tiveram melhor distribuição da lâmina do que os aspersores $\mathrm{B}$, devido ao seu menor bocal secundário desses últimos. 
TABELA 10. Resultado do teste $\mathrm{F}$ e do teste de Tukey para a vazão média dos aspersores e para os valores de CUC e de CUD, em relação aos tratamentos utilizados.

\begin{tabular}{ccllll}
\hline Tratamentos & Vazão dos Aspersores $\left(\mathrm{L} \mathrm{h}^{-1}\right)$ & $\mathrm{CUC}(\%)$ & $\mathrm{CUD}(\%)$ & $\mathrm{CUCa}(\%)$ & $\mathrm{CUDa}(\%)$ \\
\hline $\mathrm{T} 1$ & $1.875,0 \mathrm{a}$ & $90,14 \mathrm{a}$ & $80,13 \mathrm{a}$ & $93,62 \mathrm{a}$ & $89,20 \mathrm{a}$ \\
$\mathrm{T} 2$ & $1.839,6 \mathrm{a}$ & $87,88 \mathrm{ab}$ & $76,33 \mathrm{ab}$ & $91,39 \mathrm{bc}$ & $86,37 \mathrm{bcd}$ \\
T3 & $1.829,6 \mathrm{a}$ & $85,91 \mathrm{bc}$ & $75,98 \mathrm{bc}$ & $89,46 \mathrm{~d}$ & $85,96 \mathrm{~d}$ \\
T4 & $1.815,9 \mathrm{a}$ & $85,62 \mathrm{bc}$ & $76,18 \mathrm{~b}$ & $88,63 \mathrm{~d}$ & $86,54 \mathrm{~cd}$ \\
T5 & $1.685,3 \mathrm{~b}$ & $86,59 \mathrm{~b}$ & $75,31 \mathrm{bc}$ & $91,86 \mathrm{ab}$ & $88,45 \mathrm{ab}$ \\
T6 & $1.483,9 \mathrm{c}$ & $83,75 \mathrm{~cd}$ & $72,09 \mathrm{~cd}$ & $91,33 \mathrm{bc}$ & $88,22 \mathrm{abc}$ \\
T7 & $1.522,3 \mathrm{c}$ & $83,56 \mathrm{~cd}$ & $72,85 \mathrm{bcd}$ & $89,69 \mathrm{~cd}$ & $88,03 \mathrm{abc}$ \\
T8 & $1.455,2 \mathrm{c}$ & $81,76 \mathrm{~d}$ & $70,53 \mathrm{~d}$ & $89,27 \mathrm{~d}$ & $86,99 \mathrm{bcd}$ \\
\hline F & $55,9^{*}$ & $22,91^{*}$ & $12,12^{*}$ & $17,86^{*}$ & $7,46^{*}$ \\
DMS & 106,6 & 2,50 & 3,91 & 1,79 & 1,83 \\
C.V. & $3,42 \%$ & $1,58 \%$ & $2,83 \%$ & $1,07 \%$ & $1,13 \%$ \\
\hline
\end{tabular}

* significativo a 5\% de probabilidade; médias seguidas de letras distintas na mesma coluna diferem entre si, a 5\% de probabilidade.

\section{CONCLUSÕES}

O coeficiente de uniformidade de Christiansen (CUC) foi sempre maior que o coeficiente de uniformidade de distribuição (CUD), e os valores desses coeficientes decresceram com o aumento da dose de água residuária de suinocultura (ARS) até $150 \mathrm{~m}^{3}$ há $^{-1}$ ano $^{-1}$, indicando que a dose de ARS tem influência na uniformidade de distribuição.

Com o aumento da dose de ARS, o aspersor com um bocal de menor diâmetro apresentou maiores reduções de vazão e de uniformidade de distribuição, por ser mais suscetível ao entupimento.

Mesmo para a dose de $200 \mathrm{~m}^{3} \mathrm{ha}^{-1}$ ano $^{-1}$ de ARS, os valores de CUC e de CUD foram superiores aos valores mínimos recomendados para irrigação por aspersão.

\section{REFERÊNCIAS}

CHRISTIANSEN, J.E. Irrigation by sprinkling. Berkley: University of California, 1942. 124 p.

COTRIM, C.E. Análise de uniformidade de aplicação de água em sistema de irrigação do tipo Pivô Central de baixa pressão. 1988. 114 f. Dissertação (Mestrado em Engenharia Agrícola) Universidade Federal de Viçosa, Viçosa - MG, 1988.

DRUMOND, L.C.D; FERNANDES, A.L.T. Irrigação por aspersão em malha. Uberaba: Ed. Universidade de Uberaba, 2001. 84 p.

FRIZZONE, J.A. Irrigação por aspersão: uniformidade e eficiência. Piracicaba: ESALQ, Departamento de Engenharia Rural, 1992. 53 p.

HART, W.E. Subsurface distribution of uniformly applied surface waters. Transactions of the $A S A E$, St Joseph, v.15, n.4, p.656-61, 1972.

MERRIAN, J.L.; KELLER, J. Irrigation System Evaluation. A Guide for Management. Logan: Utah State University, 1978. 271 p.

PAIVA, J.B.D. Uniformidade de aplicação de água, abaixo da superfície do solo, utilizando irrigação por aspersão. 1980. 333 f. Dissertação (Mestrado em Engenharia) - Escola de Engenharia de São Carlos, Universidade de São Carlos, São Carlos, 1980.

PEREIRA, G.M. Simulação das perdas de água por evaporação e uniformidade de distribuição por aspersão. 1995. 125 f. Tese (Doutorado em Engenharia Agrícola) - Universidade Federal de Viçosa, Viçosa MG, 1995. 
PORTO, R. de M. Hidráulica básica. 2. ed. São Carlos: EESC - USP, 2000. 540 p.

RESENDE, R. Desempenho de um sistema de irrigação Pivô Central quanto à uniformidade e eficiência de aplicação de água, abaixo e acima da superfície do solo. 1992. 86 f. Dissertação (Mestrado em Agronomia) - Escola Superior de Agricultura "Luiz de Queiroz", Universidade de São Paulo, Piracicaba, 1992.

SILVA, W.L.C. Considerações sobre o uso de sprays de baixa pressão em pivô central. Revista Item, Brasília, v.39, n.12, p. 26-7, 1989.

SOIL CONSERVATION SERVICE. National Engineering Handbook. Washington: Sprinkler Irrigation, 1968. Section 15, Chapter $11.83 \mathrm{p}$.

TOOYAMANI, K.P.; NORUM, D.I.; DUBETZ, S. Application rates and uniformity under centerpivot sprinkler irrigation systems using spray nozzles. Canadian Agricultural Engineering, Ottawa, v.29, n.2, p.149-50, 1987.

WOODWARD, G.D. Sprinkler irrigation. New York: Sprinkler Irrigation Association, 1959. 377 p. 